\title{
Sistematización de una Experiencia de Inclusión en Educación Secundaria: Estrategia de Formación y Acción Educativa
}

\section{Systematization of an Experience of Inclusion at the Junior High School: Strategy for Training and Educational Action}

\author{
Juan Andrés Elías 1, * \\ Beatriz Anguiano-Escobar ${ }^{1}$ \\ Diana Irasema Cervantes ${ }^{1}$ \\ Rosario Ramírez-Bueno ${ }^{2}$ \\ ${ }^{1}$ Universidad Autónoma de Ciudad Juárez, México \\ ${ }^{2}$ Servicios Educativos del Estado de Chihuahua, México
}

\begin{abstract}
En este documento se comparte el proceso de sistematización de la incorporación de una Unidad de Servicios de Apoyo a la Educación Regular (USAER), a un centro escolar del nivel de educación secundaria. El equipo de investigación se propuso comprender la perspectiva de las personas que conforman la comunidad escolar, en torno al desarrollo de una cultura de inclusión educativa, en el marco de una investigación con enfoque participativo. Para la recuperación de la información se implementaron diversas técnicas cualitativas. Los resultados obtenidos permitieron comprender los aciertos y logros alcanzados, así como las problemáticas que se presentaron y los desafíos que permanecen; además, dicha experiencia hizo posible comprender el potencial de la sistematización de experiencias, como una estrategia formativa para la construcción colectiva de saberes, así como para la formación docente en una perspectiva participativa, y el desarrollo de proyectos integrales de acción educativa. Se concluye que es necesario desarrollar pautas que orienten el trabajo de la USAER para el nivel específico de secundaria, así como proyectos compartidos por toda la comunidad escolar, que promuevan la concientización, participación y toma de decisiones colectivas, con objeto de consolidar una verdadera cultura de inclusión educativa y de atención a la diversidad.
\end{abstract}

Descriptores: Formación de profesores; Educación especial; Acceso a la educación; Educación básica; Investigación participativa.

This document shares the process of systematizing of the incorporation of a Regular Education Support Services Unit (USAER, for its acronym in Spanish) into a junior high school. The research team set out to understand the perspective of the people who make up the school community, around the development of a culture of educational inclusion, within the framework of a research with a participatory approach. Were implemented various qualitative techniques for the recovery of information. The results obtained made it possible to understand the successes and achievements, as well as the problems that arose and the challenges that remain in view. In addition, this experience made it possible to understand the potential of the systematization of experiences, as a formative strategy for the collective construction of knowledge and the development of integral projects of educational action. Findings indicate that there is a need to develop guidelines for the work of the USAER, for the specific junior high school level, as well as projects shared by the entire school community, which promote awareness, participation and collective decision-making, in order to consolidate a true culture of educational inclusion and attention to diversity.

Keywords: Teacher education; Special education; Access to education; Basic education; Participatory research.

*Contacto: andres.elias@uacj.mx

Recibido: $18 / 06 / 2021$

$1^{\text {a }}$ Evaluación: 24/09/2021

ISSN: 0718-7378

Aceptado: 04/10/2021

www.rinace.net/rlei/ 


\section{Introducción}

La escuela pública mexicana constituye un holograma que refleja la enorme diversidad sociocultural y la acentuada desigualdad económica que prevalece en el país. Frente a esta compleja realidad, el desarrollo de experiencias educativas inclusivas y libres de Barreras para el aprendizaje y la participación (BAP), en especial para estudiantes que presentan alguna discapacidad o necesidad educativa acentuada, es un reto que permanece latente en el Sistema Educativo Mexicano y para el cual no existe, hasta el momento, una respuesta satisfactoria.

En nuestro país, la cultura escolar está aún lejos de incorporar la inclusión educativa como un principio rector de sus procesos cotidianos, inclusive en los centros escolares que cuentan con servicios de apoyo orientados a esta finalidad; esto contribuye a que los porcentajes de asistencia a la escuela de las y los estudiantes con discapacidad, sean limitados y decrezcan con el ascenso a cada nivel educativo (Secretaría de Educación Pública [SEP $]$, 2017).

Desde 1993, fecha en que se firma el Acuerdo Nacional para la Modernización de la Educación Básica, la atención al estudiantado con discapacidad se realiza, principalmente, a través de dos tipos de servicio, los Centros de Atención Múltiple (CAM) a los cuales asisten de forma regular y son atendidos por especialistas, y las Unidades de Servicios de Apoyo a la Educación Regular (USAER) que atienden al 90\% del alumnado matriculado, y en las cuales personal especializado acude a las escuelas regulares con objeto de apoyar y brindar herramientas para que las instituciones se conviertan en espacios inclusivos (Instituto Nacional para la Evaluación de la Educación [INEE], 2018).

A los CAM asisten estudiantes "con discapacidad intelectual, visual, auditiva, motriz, psicosocial, múltiple o con trastornos generalizados del desarrollo [ ...] que requieren de apoyos permanentes y especializados" (SEB, 2018, p. 9) y ajustes significativos al currículo. Las USAER por su cuenta, están dirigidas a las escuelas de educación regular que han integrado estudiantes con "discapacidad, aptitudes sobresalientes, dificultades severas de aprendizaje, de conducta o de comunicación, o con trastornos del espectro autista" (SEB, 2018, p. 17), y con quienes se puede trabajar mediante ajustes razonables.

Generalmente el trabajo de las USAER se desarrolla por parte de equipos interdisciplinarios conformados por especialistas en psicología, trabajo social, comunicación y educación especial; estos equipos atienden alrededor de cinco escuelas regulares, brindando apoyo psicopedagógico y orientación para la realización de adecuaciones curriculares dirigidas a estudiantes con Necesidades Educativas Especiales [NEE] (Romero y García, 2013). Dichos servicios de apoyo se orientan a desarrollar aulas inclusivas con el propósito de eliminar o disminuir las BAP en la escuela.

Respecto a la cobertura nacional de las USAER en el nivel educativo básico, existen considerables deficiencias; durante el ciclo escolar 2016-2017, el 71,3\% del alumnado que asistían a estas unidades correspondían al nivel de educación primaria, le seguía la educación preescolar con el 16,4\%, y finalmente, la secundaria con un 12,3\% (INEE, 
2018)1. Lo que permitía inferir que la interrupción de los estudios de estudiantes con discapacidad, es significativa en el nivel de secundaria, y que quienes llegan, no cuentan con suficientes servicios de apoyo.

En el caso de Ciudad Juárez, espacio que alberga esta investigación, el impulso a la incorporación de las USAER al nivel secundario se realizó durante el ciclo escolar 20152016, a partir de la indicación del Departamento de Educación Especial; durante este primer ciclo se trabajó en seis centros escolares federalizados² ${ }^{2}$

Para las personas que integran los equipos de la USAER este hecho significó un reto de enormes dimensiones, pues se enfrentaron a un contexto educativo distinto a los que histórica y normativamente estaban habituadas, los documentos normativos y la experiencia de los equipos se ubicaban y limitaban a los niveles de preescolar y primaria; este contexto les requirió desarrollar nuevos saberes y habilidades, y reestructurar sus procesos organizativos y de acompañamiento, además de poner en juego sus convicciones y principios educativos.

Ante este panorama, en el que incursionaron en un nivel educativo distinto, se les presentaron algunas interrogantes: ¿cómo ha sido la experiencia de incorporación de las USAER al nivel de educación secundaria en Ciudad Juárez?, ¿cuáles han sido los principales retos y dificultades que surgieron durante la incorporación?, ¿qué saberes han construido las comunidades educativas durante esta experiencia?, y a partir de esto, ¿de qué manera las USAER deben adecuar su finalidad, propósitos, estrategias, operación y recursos, con objeto de responder a las condiciones de la educación secundaria? La presente investigación asumió el reto de ofrecer algunas respuestas a tales interrogantes, a través de un proceso de sistematización de experiencias educativas, enfatizando su valor heurístico para la formación de docentes y de equipos de trabajo de USAER, así como para el establecimiento de algunas bases para el desarrollo de proyectos colectivos orientados al fomento de una cultura de inclusión y atención a la diversidad, aspecto fundamental para el desarrollo de una verdadera inclusión educativa (Rivas-Arenas, 2019).

\section{Revisión de la literatura}

La inclusión educativa es definida por Booth y Ainscow (2015), como un enfoque estrechamente vinculado con la democracia, orientado a la mejora de la experiencia educativa y de la vida social, y fundado en valores estructurales, como "igualdad, participación, comunidad, respeto a la diversidad, y sostenibilidad” (p. 25); valores referidos a las relaciones entre las personas: respeto a la diversidad, no violencia, confianza, compasión, honestidad y valor; y relacionados con la formación del espíritu: alegría, amor, esperanza/optimismo y belleza (expuestos en la página 26 del mismo texto).

\footnotetext{
${ }^{1}$ En México la educación obligatoria se conforma de los niveles de educación inicial (o a 3 años), preescolar -3 a 5 años-, primaria -6 a 11 años-, secundaria -12 a 16 años- y bachillerato -de 16 años en adelante, dependiendo del tipo de servicio-(CPEUM, 202 1; INEE, 2019).

${ }^{2}$ El sistema educativo mexicano se estructura bajo una compleja jurisdicción; la escuela pública secundaria, para el estado de Chihuahua, se divide en dos subsistemas, federal y estatal, ambos comparten estructura y propuesta curricular, sin embargo, el primero responde, en términos administrativos, a la federación, y el segundo atiende lineamientos propios del estado (INEE, 2019).
} 
Es necesario resaltar en su propuesta, que, desde su perspectiva, la dimensión instrumental-tecnocrática es, al menos, insuficiente para consolidar escuelas inclusivas; el cambio no provendrá únicamente de un cambio en las estrategias o en el herramental disponible, sino en la capacidad de las personas para transformar su visión del mundo, y con ello, del acto educativo.

En este sentido, la educación inclusiva es una utopía que permanece en el horizonte. Hacerla una realidad implica abrir líneas de acción en diversas dimensiones y ámbitos, dentro y principalmente fuera del Sistema Educativo Nacional (López y Manghi, 2021; SEP, 2017), que incidan en aspectos culturales y estructurales de importante relevancia. No obstante, sería autocomplaciente (Zúñiga, 2011), no reconocer que "las características y funcionamiento del sistema educativo tienen una notable influencia en la desigualdad educativa y las pautas de exclusión" (SEP, 2017, p. 23).

Dentro de los factores asociados al éxito escolar e internos a la escuela, cobran especial importancia para esta investigación, los procesos formativos, tanto de los equipos de USAER, como de los docentes y colectivos escolares, y más aún, los procesos de desarrollo de comunidades de aprendizaje (Elboj et al., 2006), que reconfiguren el pacto entre escuela y comunidad, y a partir de esto, impulsen proyectos integrales de transformación educativa.

Sin embargo, dicha formación para ser tal, habrá de trastocar nuestras creencias, en este caso educativas, constituidas en cimientos que condicionan y en algunos casos, quizá, determinan nuestras prácticas. En consecuencia, la formación, entre otros aspectos, requiere del análisis y la deconstrucción de nuestras creencias educativas (Creten y Huyghe, 2013; Jiménez et al., 2014).

Desde la perspectiva del equipo de investigación, esta formación demanda una participación activa del propio personal educativo y de las y los involucrados en la educación del estudiantado (Fassetta et al., 2017; Ferrada, 2017; López y Manghi, 2021); sin esta participación será difícil consolidar colectivos capaces de dialogar, consensuar y trabajar en conjunto a partir de la configuración de propósitos compartidos (Baute e Iglesias, 2011).

En este sentido, una alternativa para el desarrollo de procesos colectivos de comprensión y acción, es la sistematización de experiencias educativas (Cifuentes, 2016), estrategia metodológica-formativa que impulsa la participación activa de las comunidades, la recuperación de sus saberes y la construcción colectiva de nuevas y complejas formas de comprensión de las realidades educativas (Rivas-Arenas, 2019). Diversas experiencias anteriores muestran resultados positivos con el uso de este tipo de aproximaciones metodológicas, sobre todo en cuanto a su potencial para contrastar las distintas visiones de las personas involucradas (Baute e Iglesias, 2011; Martín, 2014), aspecto fundamental para la ampliación de nuestro entendimiento y el inicio de un diálogo en el que estemos dispuestos a despojarnos de las certezas que nos habitan (Vásquez, 2011).

Por otro lado, resulta fundamental contribuir a la comprensión de las condiciones en las que se desarrolla la inclusión educativa y las dificultades que se presentan en el trayecto, pues, como afirma el estudio realizado por Rosas y otros (2021), la inclusión educativa se ha sostenido más por criterios valorales que científicos, y vista desde el tamiz investigativo, su puesta en marcha "no asegura mejores efectos educativos que la escuela especial [segregadora]” (p. 69). No existen respuestas totales a situaciones complejas y diversas, como las que subsisten en cada sujeto con NEE, y continuar impulsando 
iniciativas educativas por decreto puede distraernos del desarrollo de estrategias efectivas que coadyuven al desarrollo pleno de las comunidades.

\section{Contextualización de la investigación}

La investigación se realizó a partir del ciclo escolar 2018-2019, por la iniciativa y participación de un colectivo de agentes educativos agrupados en torno a una USAER que atiende a centros escolares en el suroriente de Ciudad Juárez, una de las zonas más excluidas y vulneradas; participó también, personal directivo, docente y de apoyo de una escuela secundaria del subsistema federal y un equipo de investigación de la Universidad Autónoma de Ciudad Juárez (UACJ). Previo a la conformación de este colectivo, la USAER trabajó durante dos ciclos escolares, que constituyen el antecedente para este esfuerzo, y que de forma sucinta se describen a continuación.

En octubre de 2016, después de un proceso de comunicación y establecimiento de expectativas, se decidió brindar los servicios de la USAER en una secundaria federal que atiende alrededor de 1,900 estudiantes en cada ciclo escolar; esta escuela se conforma de una plantilla de 75 trabajadoras y trabajadores de la educación, entre los que se encuentran 52 docentes, personal directivo, administrativo y de apoyo, distribuidos en los turnos matutino y vespertino.

El ciclo escolar 2016-2017 constituyó el momento de entrada de la USAER. Durante este periodo era reducido el número de estudiantes con NEE diagnosticadas, las problemáticas identificadas eran moderadas o leves. El proceso de acompañamiento de la maestra de apoyo asignada se centró en los campos disciplinares de español y matemáticas, colaborando en: sensibilizar a la comunidad educativa sobre el propósito y las funciones de la USAER, brindar herramientas al profesorado frente a grupo para la realización de adecuaciones curriculares, asesorar a las familias sobre cómo llevar a cabo el acompañamiento de las y los estudiantes a su cargo, y trabajar directamente con el estudiantado; esta última no es una función prioritaria de la USAER, no obstante, representó un respiro para el profesorado con respecto a su carga de trabajo, lo cual facilitó que la comunidad evaluara como exitosa la incorporación de la USAER.

El ciclo 2017-2018 fue complicado para la experiencia de colaboración; por un lado, la escuela fue reconocida por la comunidad como un espacio que prestaba el servicio de la USAER, la única del sector, lo que derivó en el incremento de estudiantes con NEE, que además presentaban condiciones de mayor dificultad; por otra parte, los continuos cambios en el personal de apoyo adscrito a la unidad, ocasionaron un retroceso en la evolución del servicio. El cierre de este ciclo fue un momento coyuntural, los resultados no habían sido los esperados y los problemas se habían incrementado.

Tras estos resultados la comunidad escolar delineó varias estrategias, entre ellas se asignó a una nueva maestra de apoyo para atender a la secundaria durante el ciclo 2018-2019, bajo el compromiso de permanecer en el plantel por un periodo de tres años. Además, se diseñó un plan de intervención que incluía distintas adecuaciones en la operación y en el acompañamiento de la unidad.

El periodo 2018-2019 significó un momento importante en esta experiencia. Además del acuerdo de continuidad establecido con la dirección escolar y del plan de intervención, se tuvo un acercamiento con un grupo de investigación de la UACJ, con objeto de sistematizar la experiencia, con el propósito de comprenderla y trazar, en conjunto, rutas de acción para el cumplimiento de los propósitos compartidos. 


\section{Método}

\section{Perspectiva paradigmática}

El presente documento se centra en la etapa de sistematización de la experiencia, sin embargo, es sólo una parte de un proceso de mayor envergadura que asume la propuesta de investigación-acción participativa de Heron y Reason (2008), definida como una experiencia en la que las y los participantes colaboran de forma activa en todo el trayecto investigativo.

La sistematización de experiencias educativas se concibe como un proceso de recuperación, organización, análisis y reflexión de la información relacionada con una experiencia educativa previa, con objeto de comprenderla y de construir a partir de ésta, saberes situados y socialmente compartidos (Cifuentes, 2016). Como se puede prever, este proceso no agota su agenda en la comprensión del fenómeno, sino que considera esta etapa como un medio para construir saberes que permitan diseñar estrategias que logren la transformación de la práctica.

Técnicas de recolección y análisis de datos

Las técnicas para la recolección de la información fueron: para las y los estudiantes asignados a la USAER, entrevistas individuales y grupos focales; con las y los estudiantes no adscritos a USAER, grupos focales; en el caso de los responsables de familia de las y los estudiantes atendidos por la USAER, se realizó un grupo focal; para el cuerpo docente, se utilizaron dos técnicas: las narrativas autobiográficas y el grupo focal; y referente al personal de apoyo y directivo, se trabajó por medio de entrevistas. Todas estas técnicas se realizaron durante el primer semestre de 2019.

En el caso del equipo de USAER, se utilizaron las narrativas autobiográficas, grupo focal y talleres investigativos a través de la metodología de Marco Lógico adaptada al fenómeno educativo (Elías, 2017); en esta última técnica se incorporó a personal directivo de la secundaria. Estos talleres se realizaron durante el ciclo escolar 2018-2019 y parte de 20192020. A través de las diversas técnicas se indagó sobre las problemáticas relacionadas con la inclusión educativa, las expectativas y el proceso de incorporación de la USAER a la secundaria, los procesos comunicativos y colaborativos, las estrategias desarrolladas y los resultados obtenidos, así como el planteamiento de recomendaciones para redirigir las estrategias del colectivo.

En relación con el análisis de datos, se utilizó, por parte del equipo de investigación de la UACJ, el análisis de contenido propuesto por Bardin (1996), que incorpora la organización y explotación del material, codificación y categorización, y el tratamiento e interpretación de los resultados; no obstante, las conclusiones derivadas de este proceso constituyeron sólo el punto de partida para un análisis crítico (Rivas-Arenas, 2019), que involucró a toda la comunidad, con objeto de que conocieran las diversas perspectivas de las y los involucrados, reflexionaran, dialogaran y construyeran, en conjunto, una comprensión colectiva del objeto de estudio.

El establecimiento de criterios de validez universales para la investigación cualitativa es una aspiración inasequible, en virtud de la diversidad paradigmática que subyace a este campo; sin embargo, y de acuerdo con Mendizábal (2007), la presente investigación asume como criterios orientadores la credibilidad/autenticidad, la transferibilidad, la auditabilidad, la confirmabilidad y el empoderamiento. A partir de esto se buscó construir, 
en colectividad, saberes fundados "en las construcciones de sentido de los sujetos" (p. 92), principalmente mediante la valoración por parte de la comunidad de los puntos de llegada, validación comunicativa; así mismo, que los conocimientos construidos, puedan ser transferidos que no generalizados estadística y universalmente, a contextos con características similares y permitan la comprensión de situaciones particulares; que a lo largo de todo el trayecto investigativo se pueda auditar la sistematicidad y el rigor metodológico, y confirmar los hallazgos obtenidos; y finalmente, que haga posible a las y los miembros de la comunidad reconocer su voz, e incorporarla a la conversación colectiva en un acto de justicia cognitiva.

\section{Participantes}

Los participantes del estudio se clasifican en dos categorías, participantes activos que atendieron la invitación de sumarse al equipo de investigación, y participantes informantes que se incorporaron a través de las técnicas expuestas. Respecto al equipo de participantes activos, co-investigadores, se reconoce, además del equipo de investigación de la UACJ, la colaboración de la supervisora de zona de educación secundaria, la directiva del centro escolar, y la supervisora de la zona de educación especial, así como el equipo de la USAER y su director.

En relación con quienes fungieron como informantes, participaron un total de 25 estudiantes atendidos por la USAER, por presentar algún tipo de discapacidad, NEE, o rezago educativo severo; y 19 estudiantes no atendidos por la USAER (18 mujeres y un hombre), todos con edades entre los 12 y 15 años. También colaboraron siete madres y dos padres de familia, con hijos e hijas adscritos a la USAER, entre los 31 y los 45 años. Tres no contaban con estudios (dos hombres y una mujer), una de ellas sólo tenía educación primaria, una contaba con educación secundaria, tres tenían preparatoria, y finalmente, una contaba con estudios de licenciatura.

Respecto al personal del centro escolar, se sumaron 13 docentes (10 mujeres y tres hombres), con edades entre los 31 y 60 años, dos en sus primeros seis años de vida profesional docente, y 11 con siete años o más; respecto a la formación, cinco cuentan con licenciatura y ocho con maestría; tres poseen formación universitaria y 10 egresaron de escuelas normales. Además, una trabajadora social con 12 años de antigüedad en el cargo y formación académica de maestría; una orientadora educativa con nueve años de servicio y formación inicial y de maestría en psicología; dos subdirectores, uno de cada turno, con 19 y 28 años de antigüedad y estudios de maestría; la directora del centro escolar, con 18 años de experiencia y estudios de maestría; y finalmente, la inspectora de la zona escolar, también con estudios de maestría y una amplia experiencia en el sector educativo.

Por último, del personal de la USAER colaboraron ocho maestras, cuatro menores de 30 años, y otras cuatro entre los 31 y 50 años. De ellas, una es egresada de escuela normal con formación en educación especial, otra es trabajadora social y seis son universitarias de distintas instituciones, tres en el campo de la psicología y otras tres en intervención educativa; dos de ellas cuentan además con un posgrado en educación especial. Respecto a la antigüedad, cinco de ellas están en sus primeros tres años de servicio, las otras tres poseen mayor experiencia. Además, se recuperó la perspectiva del director de la USAER y de la supervisora de la zona de educación especial; ambos poseen amplia experiencia en el campo de la educación especial y cuentan con estudios de maestría.

Consideraciones éticas de la investigación 
La participación de cada una de las personas fue voluntaria y posterior a la lectura y firma de la declaración de consentimiento informado, en la cual conocieron los fines del estudio y la propuesta de investigación; uno de los acuerdos fue la preservación del anonimato y la confidencialidad de su participación. Como parte de los compromisos, los resultados fueron socializados y se buscó conocer su perspectiva al respecto, para efectos de realizar las adecuaciones y precisiones necesarias.

\section{Resultados}

Los resultados permitieron ver las distintas perspectivas de las y los participantes, mostraron su punto de vista en torno a la experiencia de incorporación de la USAER en el plantel educativo, y lo que significó, en el caso de los sujetos de obligación, hacer patente su responsabilidad de coadyuvar en la construcción de una escuela inclusiva.

Dichas perspectivas se presentan en dos tiempos, primeramente, se muestra una argumentación que recupera las perspectivas de cada grupo de participantes; luego, en un segundo momento se encuentra un análisis que cruza las distintas visiones a través de categorías comunes. Este análisis fue realizado a través del taller investigativo, constituido por el personal directivo de la secundaria, parte del equipo de la USAER, y el equipo de investigación de la universidad. Para quienes participaron significó una experiencia de diálogo, reflexión y negociación de los puntos de llegada, actividades todas que, en conjunto, constituyen una experiencia de formación y desarrollo de propuestas de acción colectiva.

\subsection{Perspectiva de las y los estudiantes de la USAER}

Las y los estudiantes adscritos a la USAER aprecian la atención personalizada que reciben por parte de la unidad; dicha atención, constituye un espacio de laboriosidad que disfrutan y en el cual construyen aprendizajes; al mismo tiempo, participar en grupos de tutorías personalizadas les hace sentir aislamiento y estigmatización, situación que se refuerza por parte de docentes que no consideran pertinente su asistencia a las sesiones de trabajo con la USAER.

En este contexto dos aspectos llaman la atención, el primero es que las y los estudiantes no conciben que las dificultades que presentan en su desempeño académico sean debido a "barreras para el aprendizaje y participación”; lejos de esta concepción, asumen su situación como el resultado de una condición personal deficiente, han incorporado que ellas y ellos son el problema, y no la escuela que es incapaz de responder ante el reto de la diversidad.

Vengo al USAER porque no sé trabajar muy bien, porque me faltan más ganas y porque no sé tanto de matemáticas. (Estudiante adscrito a USAER 1, entrevista, 2019)

Por otro lado, es necesario mencionar que en este grupo de estudiantes se ha incorporado alumnado con rezago educativo derivado de una escolarización fallida y no de características personales; esta condición es el resultado de su paso por una escuela incapaz de hacer posible una "educación de calidad", una escuela "discapacitante" que viene a incrementar el trabajo de las USAER y traslada la responsabilidad de la escuela al individuo.

\subsection{Perspectiva de estudiantes no adscritos a la USAER}


El estudiantado no adscrito a la USAER reconoce que hay compañeras y compañeros que tienen dificultades para aprender y que no reciben la atención necesaria; en contraparte, manifiesta que una parte de quienes sí están recibiendo atención, no aprovechan el apoyo y muestran una actitud apática, escudándose en su condición para obtener concesiones en cuanto al trabajo académico.

Asimismo, exponen que hay una aceptación e inclusión de compañeras y compañeros con NEE, por parte de la mayoría de las personas que conforman la comunidad escolar, y que han observado las adecuaciones y ajustes por parte de docentes, así como el compromiso de estudiantes que apoyan a quienes lo requieren. Expresan que sí han visto mejoras en el aprendizaje de sus compañeras y compañeros a partir del trabajo de la USAER y que tratan de colaborar en lo que les es posible.

Cuando nos ponen trabajos, después de que terminan de explicarnos, le hablan a él [estudiante con NEE] y le explican en el escritorio, les toma más tiempo para que entienda bien. Nosotros nos llevamos muy bien con él, de hecho, lo apoyamos mucho y cuando hacemos actividades en equipo lo ayudamos y le explicamos paso a paso lo que se tiene que hacer. (Estudiante no adscrito a la USAER 1, grupo focal, 2019)

Finalmente, resulta esperanzador que tales estudiantes sugieren se les dé a conocer la forma de trabajo de la USAER para que así puedan apoyar más; esto abre posibilidades para la incorporación de estrategias en que toda la comunidad no sólo sea sensible a la diversidad, sino que coadyuve de forma activa:

\begin{abstract}
To quisiera saber cuál es el modo de trabajo hacia los niños de USAER, porque me gustaría poder apoyarlos; [...] saber qué es lo que hacen para cambiar su disciplina, porque van muy enojados o a veces llorando, y regresan con una sonrisa. (Estudiante no adscrito a la USAER 2 , grupo focal, 2019)
\end{abstract}

\title{
4.3. Perspectiva de tutoras y tutores legales del estudiantado vinculado a la USAER
}

La participación de las familias en el proceso educativo de las y los estudiantes a su cargo ha estado permanentemente en el foco de la discusión, situación que en los últimos años se ha recrudecido y que requiere un abordaje multirreferencial que se escapa a las posibilidades de este documento. No obstante, se puede afirmar que los cambios en las dinámicas de trabajo y socialización hacen cada vez más necesario repensar el rol de las y los tutores legales y mejorar el acompañamiento que realizan; este interés incluso se ha objetivado en acuerdos secretariales y recientemente en la modificación al artículo tercero constitucional.

Las familias, como resultado de la mediación e intervención del equipo de USAER, expresan conocimiento sobre las funciones de la unidad, y enfatizan la asesoría que reciben para realizar un mejor acompañamiento de las y los estudiantes a su cargo. En general la valoración sobre la USAER es favorable, perciben un avance considerable en el desempeño académico del alumnado adscrito, lo que se refleja en un mejor ánimo e implicación escolar.

\footnotetext{
Mi experiencia en lo general es de agradecimiento por que existan docentes que ayuden a jóvenes con necesidades diferentes. A mí me han ayudado a que mi hïo tenga una oportunidad de seguir sus estudios, [...] a manejar situaciones que pasan, me apoyan con ciertas conductas de mi hijo, me orientan. (Responsable de familia 2 , narrativa autobiográfica, 2019)
}

Respecto al clima escolar, aun cuando se mencionan casos de discriminación o acoso, se cree que estos son aislados, y se percibe un ambiente de respeto hacia el estudiantado con NEE, tanto por parte del cuerpo docente, como por parte de la comunidad estudiantil; 
reconocen un cambio en la actitud del profesorado, al cual se le observa más informado y sensibilizado.

Las deficiencias que exponen tienen que ver con el número limitado de personal de la USAER, dada la cantidad de estudiantes que requieren su ayuda; además, se menciona que una parte del profesorado no apoya el proceso de inclusión, ni el desempeño académico favorable de las y los estudiantes que atiende la USAER, ya sea desde su propia práctica, o en vinculación con la unidad.

Creo que las dificultades que hay, es por falta de maestras. Dos maestras no son suficientes para toda la escuela, a los estudiantes no se les da la atención que se necesita, no hay comunicación con todos los maestros y en ocasiones con el mismo padre de familia. (Responsable de familia 5 , narrativa autobiográfica, 2019)

Es importante tomar en cuenta que, en la elección de la escuela, las familias sí consideran como un criterio fundamental la presencia de una USAER, por esta razón se concibe importante planificar la entrada de las unidades a los planteles, de forma que la transición a la inclusión educativa no sea más problemática de lo que ya es, pues al aumentar el número de estudiantes que requieren el servicio, es necesario que haya recursos suficientes para brindarlo de forma pertinente.

Por último, se resaltan tres aspectos: la USAER, con todas las dificultades que pueda enfrentar para realizar su trabajo y las deficiencias que se identificaron, representa un apoyo invaluable para las familias; éstas, consideran que las capacidades de las y los estudiantes a su cargo son limitadas, por lo cual pugnan por una educación diferenciada; y la participación de las familias se da bajo una lógica subordinada en la que siguen las instrucciones del equipo de la USAER, sin colaborar de manera colectiva u horizontal en la toma de decisiones.

\subsection{Perspectiva de docentes}

Las y los docentes son actores centrales en el proceso educativo, en ellas y ellos descansa la posibilidad de catalizar los recursos que la sociedad invierte en la educación, y transformarlos en oportunidades para construir aprendizajes. Pueden también ser el principal obstáculo si sus actitudes, conocimientos y capacidades no están a la altura del reto que se nos presenta.

El sentimiento generalizado del profesorado del plantel de secundaria, es de desencanto, a partir de que la USAER no respondió a sus expectativas iniciales. Dichas expectativas no se fundan en el modelo de inclusión educativa mexicano, ni en la capacidad organizativa y la normatividad sobre las que trabaja la USAER, pero esto no es percibido por todo el profesorado.

Cuando llega la USAER a la escuela sentí una tranquilidad porque mi idea era que ellas trabajarían con los alumnos NEE (que las maestras se los llevarían a un salón y sería un trabajo personalizado), pero no fue así. (Maestra de grupo 9, narrativa autobiográfica, 2019)

La incorporación de la unidad a la escuela se percibe adicionalmente como un problema por la tendencia a atraer a estudiantes con NEE. En este sentido se perciben posicionamientos sociológicos funcionalistas (Martínez, 2014) que no abonan al desarrollo de una escuela incluyente y justa. No obstante, sus planteamientos tienen una base empírica que deberá ser atendida, en virtud de las limitantes que el modelo inclusivo, y la USAER en lo particular, tienen de forma inherente. Lo anterior se puede ilustrar a través de las palabras de una de las docentes participantes: 
Realmente el programa de USAER no me ha dado las armas que necesito para llevar a cabo con éxito mi trabajo como docente, pues los alumnos no están respondiendo favorablemente, [...] trabajar con 42 alumnos más los niños con NEE es bastante desgastante. Pienso que todo esto no ha dado resultado, porque USAER solo trabajaba en las escuelas primarias, un solo maestro con un grupo reducido. En la escuela secundaria somos varios maestros con grupos numerosos, 50 minutos de horaclase. Esto es algo parecido a ensayo-error, de esta forma estamos trabajando. (Maestra de grupo 1, narrativa autobiográfica, 2019)

Adicional a esta perspectiva, el profesorado valora el esfuerzo realizado por la USAER, además de los apoyos que han recibido para la mejora de su práctica educativa; manifiestan deseos de continuar trabajando de manera colaborativa y seguir explorando formas diversas de educar.

\begin{abstract}
El personal de apoyo de USAER siempre ha estado con la mejor disposición de atender las propuestas e iniciativas que se les han planteado, con el tiempo que han estado se ha ido re-direccionando el camino a seguir, y paso a paso se ha ido mejorando, en la medida de las posibilidades, tanto de ellos como equipo especialista en el tema, así como de nosotros. (Maestro de grupo 11, narrativa autobiográfica, 2019)
\end{abstract}

\title{
4.5. Perspectiva del personal asignado a trabajo social y orientación educativa
}

Este personal manifiesta su deseo de colaborar de manera activa en el proyecto inclusivo, que considera estrechamente ligado a sus funciones sustantivas. Sin embargo, mencionan que las diferentes labores que desempeñan dificultan el trabajo que tienen que llevar a cabo para los casos de la USAER. Pese a esta carga de trabajo, manifiestan que siempre buscan colaborar de manera cercana y activa.

Estas figuras educativas declaran que el trabajo directo con cada joven por parte de la USAER reflejaría mayor avance en el aprendizaje, aspecto que se contrapone con las orientaciones normativas de la unidad. Destacan también, como dificultades, que las familias no se involucran lo suficiente y que el profesorado sigue sin saber exactamente cómo trabajar con las y los diferentes estudiantes con NEE:

No hay tiempo de calidad de los padres con los alumnos, todo el día trabajan para
poderlos mantener [...]. Hay muchas familias divorciadas, [...] falta de valores y
atención. [...] De los alumnos de educación especial que tengo son pocos los que tienen
padres que realmente se preocupen, que les busquen un diagnóstico, que les den un
medicamento. (Orientadora, entrevista, 2019)

\subsection{Perspectiva de personal adscrito a la subdirección}

Las y los subdirectores de gestión tienen la responsabilidad de apoyar en la organización escolar, su perfil profesional les demanda conocer el funcionamiento de la escuela y las características del trabajo en el aula. Se plantea que, a partir de su liderazgo, se oriente al personal y a la comunidad educativa en general, para que se desarrolle un entorno que apoye el aprendizaje, lo cual incluye conocer y atender las BAP y fomentar las acciones para superarlas.

Los subdirectores, ambos son hombres, coinciden en señalar como dificultades que la escuela cuenta con alumnado que requiere una atención especializada, además de una gran cantidad de estudiantes que tienen rezago académico. Asimismo, señalan que algunas de estas y de estos alumnos no están atendidos convenientemente en su hogar y que en el contexto escolar los grupos numerosos dificultan al profesorado brindar una atención suficiente a estudiantes con NEE, ya que tienen la presión de cumplir con las demandas de logro para las y los demás estudiantes. También ven como dificultad las expectativas 
que tenían acerca de la forma de trabajar de la USAER, pues asumieron que atenderían de forma personalizada a las y los estudiantes, pero en lugar de ello, se asesora a docentes para que brinden el apoyo, lo que, si bien comprenden, consideran es insuficiente.

Manifiestan que la gran mayoría del personal está comprometido con la mejora educativa y que han realizado un esfuerzo para atender a las y los alumnos, aun antes de que llegara la USAER; destacan que han intentado ajustarse a la forma de trabajo de la unidad. Igualmente, señalan que el profesorado y el equipo de USAER trabajan arduamente, que se realizan evaluaciones y seguimiento al estudiantado, que se brinda capacitación a las y los maestros y se les orienta sobre cómo realizar su práctica educativa.

En cuanto a errores y deficiencias, destacan que el personal de USAER es poco, y no está de forma permanente en la escuela, lo que impide un seguimiento personalizado y un trabajo más constante con el alumnado que les requiere. Desde su perspectiva, la forma de trabajo de la USAER genera más trabajo y presión para el personal docente, ya que deben responder a exigencias de múltiples figuras, sin que reciban un apoyo eficiente, ya que todo el trabajo fuerte deben hacerlo ellas y ellos.

\subsection{Perspectiva de directiva y supervisora}

La gestión escolar es una parte determinante en el funcionamiento de una escuela. Bajo las formas y dinámicas del sistema educativo mexicano esta labor descansa en las y los directivos, quienes tienen la autoridad para movilizar a sus respectivos colectivos. En el caso de esta escuela secundaria, el impulso hacia la cultura de la inclusión educativa tiene en las directivas parte de su explicación. Ambas, supervisora y directiva, se muestran comprometidas y convencidas, de que aún con las deficiencias presentadas, la escuela debe seguir su camino hacia la inclusión educativa.

Su posición de bisagra busca mediar entre las condiciones de la práctica docente y su legítimo reclamo por un modelo de inclusión más eficiente, y la estructura normativa y capacidad organizativa de la USAER, con las deficiencias inherentes. Su labor de impulso hacia el trabajo docente hizo viable el trabajo realizado hasta ahora en cuanto a inclusión, por otro lado, la comunicación con el equipo de educación especial ha impulsado a la unidad a mejorar sus procesos y a realizar adaptaciones con objeto de atender las necesidades del colectivo escolar.

\subsection{Perspectiva de equipo USAER}

El equipo enuncia, como dificultades, los tiempos con lo que se cuenta para realizar ciertos procesos de la USAER, pues son limitados y diferentes al contexto de la escuela primaria, en el que habían venido trabajando; además de que los grupos son numerosos, el trabajo con las familias de estudiantes es difícil, no se realiza un proceso de evaluación con el profesorado para comprender cómo están evolucionando o si les sirve lo que se les ofrece, aunado a una actitud de resistencia por parte de algunas y algunos docentes. Argumentan que,

no siempre se obtiene respuesta positiva y actitud, así como entendimiento de los casos individuales de los alumnos por parte de los docentes, sin embargo, es en ese enfrentamiento en el que el trabajo debe de ser realizado a través de los tiempos establecidos en las cargas administrativas, falta de disposición para realizar ajustes necesarios de manera conjunta, tiempo limitado y poca atención en los informes mensuales expuestos en los consejos técnicos escolares. (Maestra USAER 4, narrativa autobiográfica, 2019) 
Como fortalezas mencionan que, en lo general, la escuela está comprometida con la diversidad y la inclusión, hay disposición de las familias y de parte del personal docente para involucrase y trabajar, además, es una escuela que continuamente está desarrollando múltiples proyectos, lo que enriquece la vida escolar. Entre los aciertos o logros, se encuentra la importancia que le otorgan a mantener la comunicación entre las personas involucradas en el proceso, la búsqueda por mejorar la forma de comunicarse y apoyar al profesorado de la escuela, así como la relevancia que tienen los talleres para estudiantes con NEE y cómo es que estos les brindan un espacio seguro o de pertenencia.

Respecto a las deficiencias, enuncian que el personal docente reclama un acompañamiento prescriptivo que facilite su tarea:

La primera barrera a la que me enfrenté fue de tipo actitudinal por parte de los docentes regulares, pues mostraron inconformidad con la forma en la que venía trabajando la USAER, expresaban su descontento y la opinión de que no se les estaba brindando el apoyo en la forma en que ellos esperaban. (Maestra USAER 1, narrativa autobiográfica, 2019)

Aunado a esto, detectan como necesidades o tareas pendientes, replantear el quehacer de la USAER, sensibilizar a las y los docentes sobre el aprendizaje y ritmo distinto de cada estudiante, evaluar los procesos de aprendizaje del magisterio, así como involucrarles en todo el proceso.

\subsection{Cruce de perspectivas}

Con base en el análisis previo se realizó un cruce de perspectivas con las categorías obtenidas en el análisis de la información, a través del cual se percibieron similitudes en distintos cruces, y se pudo construir, a partir de las diferencias, realidades complejas nutridas por las distintas subjetividades consustanciales a la posición de las y los actores educativos involucrados.

\section{En algunas de las participaciones que he leído hay un mundo diferencia, entre cómo nosotros pensamos que estamos bien, que estamos ayudando y que estamos encaminando, y al leer las perspectivas de otros, expresan que no es así, que en realidad el trabajo no ha sido suficiente, que es muy poco lo que hemos ayudado. (Maestra USAER 2, taller investigativo, 2019)}

Este abordaje hizo posible observar que las dificultades, una vez asumidas, pueden ser generadoras de alternativas de acción, y que el compromiso de cada actor, así como el apoyo y trabajo colectivo, abren vías esperanzadoras.

En cuanto al reconocimiento de errores y deficiencias, se destaca la necesidad de realizar planes de trabajo sistemáticos, pero, sobre todo, participativos y sensibles a las distintas perspectivas y necesidades de cada persona involucrada. En este tenor, una comunicación efectiva, afectiva y permanente, resulta fundamental para construir comunidades que trabajen en conjunto. Es importante destacar a su vez, que la incorporación de la USAER a la escuela secundaria es un fenómeno relativamente reciente en nuestro contexto, y que en este sentido habrá de concebirse como una etapa experimental de la cual devendrán ajustes y adecuaciones.

De lo relacionado a los aciertos o logros, se puede exponer que las familias, salvo algunas excepciones, han respondido de forma favorable, ayudando en casa y realizando el seguimiento correspondiente; el equipo de la USAER ha realizado procesos que permiten un avance en los diferentes casos, así como el que las y los estudiantes beneficiarios se muestren satisfechos y entusiasmados; el personal directivo y el colectivo docente se han 
ido sensibilizando e incorporando a los procesos establecidos en el modelo de inclusión mexicano, y a la par, han hecho evidentes sus vacíos e inconsistencias, lo que habrá de servir para construir una verdadera transformación educativa que haga realidad la educación inclusiva.

Sobre los aprendizajes, se destaca que tanto el alumnado no atendido por la USAER, como los que se han incorporado a la unidad, se han adaptado bien, estos últimos tienen avances visibles. En general, se percibe una comunidad que avanza hacia la inclusión y que construye mayores recursos para la atención a la diversidad.

Creo que el trabajo que la USAER realiza, aunque está lejos de ser perfecto, ha
proporcionado a los maestros algunas herramientas valiosas que facilitan el trabajo y
la atención de los jóvenes con NEE, [...] hemos visto con agrado que las sugerencias
de los maestros se han tomado en cuenta; falta trabajo por hacer, pero considero que
vamos por buen camino, con todo y que es dificil la atención y la integración de los
alumnos con NEE en el contexto de educación secundaria. (Maestro de grupo 6 ,
narrativa autobiográfica, 2019)

Por último, en cuanto a las tareas por realizar, queda pendiente un mayor y mejor acompañamiento al profesorado, lo que incluye desarrollar procesos formativos más pertinentes y efectivos; el desarrollo de material didáctico; la elaboración conjunta de documentos normativos que constituya un marco de trabajo contextualizado; y el logro de una mayor concientización e involucramiento de todas y todos los agentes insertos en la comunidad escolar.

\section{Discusión y conclusiones}

Este proceso de recuperación, organización, análisis y reflexión en torno a la información obtenida de la experiencia educativa permitió construir una comprensión compleja, y a partir de ésta, saberes situados y socialmente compartidos. Un primer punto de llegada consiste en enfatizar el valor del trabajo colaborativo (López y Manghi, 2021) y de los ejercicios de investigación de corte participativo — que para este ejercicio fue la sistematización de experiencias educativa (Cifuentes, 2016; Rivas-Arenas, 2019)—, en los cuales se conformen comunidades de aprendizaje (Elboj et al., 2006), que construyan alianzas con otros sectores e instituciones, en este caso particular, la universidad; comunidades ampliadas que abandonen las lógicas subordinantes e indaguen, aprendan y eduquen en y para la colectividad, medio y fin de una educación emancipadora y de una experiencia formativa en los términos que el colectivo lo concibe.

En relación con el propósito de la investigación, y como un balance general de la incorporación de la USAER a la escuela secundaria, se puede afirmar que la tarea colectiva ha sido complicada, sobre todo por la ausencia de marcos normativos contextualizados y experiencias que sirvieran como referentes en el nivel de educación secundaria. Aunado a esto, el llevar a cabo el trabajo para informar, sensibilizar y dinamizar a la comunidad escolar sobre la inclusión educativa, fue una tarea demandante, en razón de que las representaciones sociales en torno a este nivel difieren sustancialmente de los niveles anteriores; sin embargo, se coincide en que la construcción de una cultura inclusiva implica, necesariamente, la constante sensibilización de cada persona involucrada y el desarrollo de estrategias y acciones en el marco de un proyecto compartido, y desarrollado de manera sistemática y fundamentada (Rosas et al., 2021). 
La comunidad comprendió, a través de la sistematización de la experiencia, que el modelo organizativo y normativo bajo el cual trabaja la USAER en educación preescolar y primaria, es inoperante en el nivel educativo de secundaria; en este tenor es fundamental el desarrollo de un marco de trabajo contextualizado para estas comunidades, que además de ser más numerosas, poseen características que las distinguen y les implican un abordaje que cuestiona las certezas construidas a lo largo de la breve historia de la inclusión educativa mexicana.

Permanece en el ambiente la pregunta sobre el modelo general de inclusión educativa en nuestro país, pese a la actitud dispuesta de las comunidades escolares, a la formación continua de sus distintos actores, y a los esfuerzos individuales y colectivos, los resultados siguen siendo insuficientes y el impacto en las y los educadores es tan abrumador, que puede llevarles a escenarios de frustración; cabe entonces la pregunta: ¿debemos continuar buscando la eficiencia de esta maquinaria que hemos construido, o quizá es momento de imaginar otras formas de enfrentar el reto de una educación integral para la diversidad?

Otro aspecto que se yergue frente al equipo de trabajo es el carácter "discapacitante" de la educación bajo el estado actual. Más allá de los pésimos resultados en pruebas estandarizadas (Backhoff et al., 2018), es necesario observar en nuestros contextos cómo la escuela va construyendo déficits que a la vuelta de varios ciclos escolares incrementan las desventajas en la población en situación de desigualdad; la escuela, en su peor faceta, no sólo reproduce la desigualdad, sino que coloca a grupos poblacionales en una situación equivalente a una discapacidad. Urge pensar y construir una escuela más justa (Zúñiga, 2011).

Al mismo tiempo resulta necesario interpelar los conceptos que acuñamos para comprender y nombrar las realidades educativas. El concepto de barreras para el aprendizaje y la participación, nos invita a descentrar nuestra mirada en una persona "distinta y de facto deficiente", y mirar a los contextos escolares como espacios incapaces de albergar a la diversidad y de responder con solvencia al reto educativo. Pero, ¿hasta qué punto utilizamos conceptos sin comprenderles, ni asumir el desplazamiento epistemológico que nos demandan?, estos conceptos corren el riesgo de constituirse en eufemismos vacuos que acoten la transformación educativa.

Este ejercicio de investigación presentó algunas limitaciones como la escasa participación del colectivo de docentes de secundaria, en cuanto a número y en relación con el tipo de colaboración, que en su mayoría se limitó a proporcionar información y a ofrecer sus opiniones sobre los distintos puntos de llegada que la comunidad activa construía en las distintas etapas; por otro lado, se hizo evidente la dificultad para el desarrollo de la participación crítica de algunas de las y los distintos actores educativos, en un sistema educativo caracterizado por su excesiva jerarquización y las significativas limitaciones administrativas que inhiben la libre comunicación de las ideas (Rivas-Arenas, 2019).

El panorama se muestra a claroscuros, con importantes avances, pero considerables vacíos. La comunidad escolar se encuentra dividida en sus percepciones, pero con la disposición suficiente para el desarrollo de estrategias conjuntas. Las y los estudiantes, principales beneficiarios de este proyecto, cuentan con colectivos aliados que día a día construyen mejores recursos para apoyarles, no obstante, concebir a la escuela como un espacio inclusivo y libre de barreras para el aprendizaje, es una meta que aún permanece en el horizonte. 


\section{Referencias}

Backhoff, E., Guevara, G., Hernández, J. y Sánchez, A. (2018). El aprendizaje al término de la educación media superior en México. El Cotidiano, 208, 7-20.

Bardin, L. (1996). Análisis de contenido. AKAL.

Baute, L. y Iglesias, M. (2011). Sistematización de una experiencia pedagógica: La formación del profesor universitario. Pedagogía Universitaria, 16(1), 36-49.

Booth, T. y Ainscow, M. (2015). Guía para la educación inclusiva. Desarrollando el aprendizaje y la inclusión en los centros escolares. OEI.

Cifuentes, R. (16-18 de noviembre de 2016). IAP y sistematización de experiencias: Apuestas, propuestas, desafíos para construir educaciones e intervenciones pertinentes y potenciadoras. Memoria académica del V Encuentro Latinoamericano de Metodología de las Ciencia Sociales (pp. 1-29). UNLP-FAHCE.

Creten, S. y Huyghe, S. (2013). Teaching at the university of Leuven: A case of teacher training in higher education in flanders. Revista de Docencia Universitaria, 11(3), 73-90. https://doi.org/10.4995/redu.2013.5521

Elboj, C., Puigdellívol, I., Soler, M. y Valls, R. (2006). Comunidades de aprendizaje. Transformar la educación. Graó.

Elías, J. A. (2017). Diseño y planificación de proyectos educativos. Una adaptación de la metodología de marco lógico al ámbito educativo. UACJ.

Fassetta, G., Grazia, M., Frimberger, K., Attia, M. y Al-Masri, N. (2017). Online teacher training in a context of forced immobility: The case of Gaza, Palestine. European Education, 49(3), 133-150. https://doi.org/10.1080/10564934.2017.1315538

Ferrada, D. (2017). Formación docente para la diversidad. RMIE, 22(74), 783-811.

Heron, J. y Reason, P. (2008). Extending epistemology within a co-operative inquiry. En P. Reason y H. Bradbury, The SAGE handbook of action research. participative inquiry and practice (pp. 366-380). Sage. https://doi.org/10.4135/9781848607934.n32

INEE. (2018). Panorama educativo en México. Indicadores del sistema educativo nacional 2017. Educación básica y media superior. INEE.

INEE. (2019). Panorama educativo de México. Indicadores del sistema educativo nacional 2018. Educación básica y media superior. INEE.

Jiménez, Y., Hernández, J. y Ortega, J. (2014). ¿Forman los programas de formación docente? $\begin{array}{lllll}\text { CPU-e, Revista Investigación } & \text { Educativa, } & 19, & 1-27 .\end{array}$ https://doi.org/10.25009/cpue.v0i19.964

López, J. y Manghi, D. (2021). ¿Es posible transformar las prácticas evaluativas mediante el trabajo colaborativo? Una mirada inclusiva a la evaluación en aula. Revista Latinoamericana de Inclusión Educativa, 15(1), 173-187. https://doi.org/10.4067/s0718-73782021000100173

Martín, D. (2014). Estrategia de formación continua dirigida a los docentes universitarios para potenciar el aprendizaje desarrollador. Revista Infociencia, 18(4), 1-10.

Martínez, R. (2014). Pedagogía tradicional y pedagogía crítica. Doble Hélice.

Mendizábal, N. (2007). Los componentes del diseño flexible en la investigación cualitativo. En I. Vasilachis (Coord.), Estrategias de investigación cualitativa (pp. 65-105). Gedisa.

Rivas-Arenas, M. (2019). La sistematización de experiencias en educación inclusiva en Colombia una alternativa de investigación. Perspectivas Educativas, 8(1), 137-147. 
Romero, S. y García, I. (2013). Educación especial en México. Desafíos de la educación inclusiva. Revista Latinoamericana de Educación Inclusiva, 7(2), 77-91.

Rosas, R., Espinoza, V., Hohlberg, E. y Infante, S. (2021). ¿Es siempre exitosa la inclusión educativa? Resultados comparativos del sistema regular y especial. Revista Latinoamericana de Educación Inclusiva, 15(1), 55-73. https://doi.org/10.4067/s0718-73782021000100055

SEB. (2018). Orientaciones generales para el funcionamiento de los servicios de educación especial 2018. SEB.

SEP. (2017). Modelo Educativo. Equidad e inclusión. SEP.

Vásquez, C. (2011). Conversemos. Revista Universidad de Antioquia, 304, 69-82.

Zúñiga, V. (2011). La escuela incluyente y justa. Antología comentada al servicio de los maestros de México. Fondo Editorial de Nuevo León-Universidad de Monterrey.

\section{Breve CV de los/as autores/as}

\section{Juan Andrés Elías}

Licenciado en Educación y Maestro en Investigación Educativa Aplicada por la UACJ; egresado del Doctorado en Ciencias de la Educación para el Centro de Investigación y Docencia (CID). Alterna la docencia y la investigación en el Departamento de Humanidades de la UACJ y en el Centro Chihuahuense de Estudios de Posgrado (CCHEP). Se ha especializado en formación, acompañamiento y evaluación de docentes de diferentes niveles educativos y en distintos aspectos del fenómeno educativo: investigación e intervención educativa, análisis multirreferencial de la práctica docente, procesos de enseñanza-aprendizaje y diseño curricular. Sus líneas de generación y aplicación del conocimiento incorporan los procesos de enseñanza-aprendizaje, sujetos de la educación y metodología de la investigación e intervención educativa. Email: andres.elias@uacj.mx

ORCID ID: http://orcid.org/0000-0002-0876-3542

\section{Beatriz Anguiano-Escobar}

Docente investigadora de la Universidad Autónoma de Ciudad Juárez, con reconocimiento del Programa para el Desarrollo Profesional Docente, para el tipo superior (PRODEP). Doctora en Pedagogía Crítica por el Instituto de Pedagogía Critica, Maestra en Educación Campo: Práctica docente e integración cultural, por la Universidad Pedagógica Nacional (UPN). Tiene experiencia en actividades de docencia, administración, intervención e investigación educativas tanto en instituciones públicas como de la sociedad civil; colabora en procesos de formación y actualización de docentes y educadores de contextos diversos, en modalidad tanto presencial como a distancia. Sus líneas de generación y aplicación del conocimiento son: políticas educativas, procesos de enseñanza-aprendizaje, sujetos de la educación. Email: beatriz.anguiano@uacj.mx

ORCID ID: http://orcid.org/OOOO-0002-3533-5607

\section{Diana Irasema Cervantes}

Doctora en Psicología por la Universidad de Guadalajara, Maestra en Derechos Humanos por la Universidad Autónoma de Ciudad Juárez (UACJ), Licenciada en Psicología por la UACJ. En la actualidad es profesora investigadora de tiempo completo en el programa de 
la Licenciatura en Educación y en la Maestría en Investigación Educativa Aplicada en la UACJ. Es formadora del Modelo educativo UACJ, así como del área de Vinculación, con la impartición de cursos a externos. Es perfil PRODEP, miembro del Sistema Nacional de Investigadores (nivel I), así como miembro del comité académico de la Maestría anteriormente mencionada. Sus líneas de investigación son: aptitudes sobresalientes y talento en jóvenes, talento docente, procesos de enseñanza-aprendizaje. Email: diana.cervantes@uacj.mx

ORCID ID: http://orcid.org/0000-0003-2353-1309

\section{Rosario Ramírez-Bueno}

Licenciada en educación primaria por la Escuela Normal Urbana, Profesor Carlos A. Carrillo, y Maestra en Educación por el Instituto Tecnológico y de Estudios Superiores de Monterrey. Se ha desempeñado como docente frente a grupo en nivel primaria, como maestra de apoyo, así como directora de la Unidad de Servicios de Apoyo a la Educación Regular (USAER); actualmente tiene la función de supervisora de Zona de Educación Especial. Aunado a estas tareas, ha fungido como instructora del Instituto Nacional para la Educación de los Adultos (INEA); docente de la Licenciatura en Educación, en la Universidad Autónoma de Ciudad Juárez (UACJ), así como evaluadora por parte de Servicio Profesional Docente, además de participar en diversas experiencias de formación continua para docentes. Email: mtrarosario7@gmail.com

ORCID ID: http://orcid.org/0000-0003-3990-1087 